\title{
On Matching Latent Fingerprints
}

\author{
Anil K. Jain, Jianjiang Feng, Abhishek Nagar \\ Department of Computer Science and Engineering \\ Michigan State University \\ East Lansing, MI-48824, U.S.A. \\ jain, jfeng, nagarabh@msu.edu
}

\author{
Karthik Nandakumar \\ Institute for Infocomm Research \\ A*STAR, Fusionopolis, Singapore \\ knandakumardi2r.a-star.edu.sg
}

\begin{abstract}
Latent fingerprint identification is of critical importance to law enforcement agencies in forensics application. While tremendous progress has been made in the field of automatic fingerprint matching, latent fingerprint matching continues to be a difficult problem because the challenges involved in latent print matching are quite different from plain or rolled fingerprint matching. Poor quality of friction ridge impressions, small finger area and large non-linear distortion are some of the main difficulties in latent fingerprint matching. We propose a system for matching latent images to rolled fingerprints that takes into account the specific characteristics of the latent matching problem. In addition to minutiae, additional features like orientation field and quality map are also used in our system. Experimental results on the NIST SD27 latent database indicate that the introduction of orientation field and quality map to minutiae-based matching leads to good recognition performance despite the inherently difficult nature of the problem. We achieve the rank-20 accuracy of $93.4 \%$ in retrieving 258 latents from a background database of 2,258 rolled fingerprints.
\end{abstract}

\section{Introduction}

Fingerprint images can be broadly classified into three categories, namely, (i) rolled/full, (ii) plain/flat and (iii) latent $[10,12,14]$ (see Figure 1). Rolled fingerprint images are obtained by rolling a finger from one side to the other ("nail-to-nail") in order to capture all the ridge-details of a finger. Plain impressions are those in which the finger is pressed down on a flat surface but not rolled. While plain impressions cover a smaller area than rolled prints, they typically do not have the distortion introduced during rolling. Rolled and plain impressions are obtained either by scanning the inked impression on paper or by using live-scan

\footnotetext{
${ }^{1}$ This research was supported by ARO grant W911NF-06-1-0418 and NIJ grant 2007-RG-CX-K183.
}

devices. Since rolled and plain fingerprints are acquired in an attended mode, they are typically of good quality and are rich in information content. In contrast, latent fingerprints are lifted from surfaces of objects that are inadvertently touched or handled by a person through a variety of means ranging from simply photographing the print to more complex dusting or chemical processing $[9,13]$. It is the matching of a latent fingerprint against a database of rolled prints or latent prints (reference prints) that is of utmost importance in forensics to apprehend a criminal.

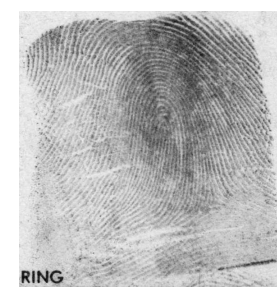

(a)

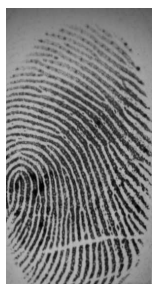

(b)

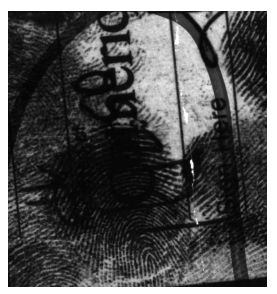

(c)
Figure 1. Three types of fingerprint images. (a) Rolled fingerprint, (b) Plain fingerprint and (c) Latent fingerprint.

Latent fingerprints obtained from crime scenes have served as crucial evidence in forensic identification for more than a century. However, there have been instances where mistakes in latent fingerprint identification have led to wrongful convictions. One of the most high profile cases in which such a mistake was made is the case of Brandon Mayfield who was wrongly apprehended in the Madrid train bombing incident after a latent fingerprint obtained from the bombing site was incorrectly matched with his fingerprint in the FBI database [5]. An extensive account of similar cases has been brought to light by the Innocence project [1]. These incidents and findings have undermined the importance of latent fingerprints as forensic evidence in courts of law. This is evident from recent ruling of a Baltimore court [6] which excluded fingerprints as evidence in a murder trial because the prosecutor was not able to justify the procedure followed in latent fingerprint matching as 
being sufficiently error free. It is clear that more research is needed to advance the state of the art in latent fingerprint matching.

The manual latent identification process can be divided into four steps, namely, (i) analysis, (ii) comparison, (iii) evaluation and (iv) verification. This process is commonly referred to as the ACE- $\mathrm{V}$ procedure in latent fingerprint literature [8].

- Analysis refers to assessing the latent fingerprint to determine whether sufficient ridge information is present in the image to be processed and to mark the features along with the associated quality information. The latent print analysis is usually performed manually by a human expert (without access to a reference print).

- Comparison refers to the stage where an examiner compares a latent image to a reference print to ascertain their similarities or dissimilarities. Fingerprint features at all three levels (Level-1, Level-2 and Level3) are compared at this stage.

- Evaluation stage refers to classifying the fingerprint pair as individualization (identification or match), exclusion (non-match) or inconclusive.

- Verification is the process of re-examination of a fingerprint pair independently by another examiner in order to verify the results of the first examiner.

It is often argued that matching a latent fingerprint to a rolled print is more of an "art" than "science" $[15,16]$ because the matching is based on subjective appraisal of the two fingerprints in question by a human examiner. Moreover, the decisions made by latent examiners are required to be "crisp", i.e., an examiner is expected to provide only one of the three decisions, viz., individualization (identification or match), exclusion (non-match) and inconclusive [9, 13]. This precludes a latent examiner to prepare a well structured and quantitative latent examination report which can be studied to estimate the chances of making an error in a particular case. Often latent examiners have a huge backlog of cases and are usually under time pressure to match latent fingerprints, particularly in high profile cases. Therefore, it is very important that the cases sent to latent examiners be efficiently selected by an automatic fingerprint matching system so that the latent examiners can spend adequate amount of time in matching them.

In order to deal with matching efficiency, the concept of "Lights-Out System" for latent matching has been introduced [10]. A Lights-Out System is characterized by a fully automatic (no human intervention) identification process. Such a system should automatically extract features from query fingerprints (latent) and match them with a gallery database (rolled or latent) to obtain a set of possible "hits" with high confidence so that no human intervention is required. But due to the limitations of the available algorithms, only "Semi Lights-Out Systems" are currently feasible especially for latent prints. In a Semi-Lights-Out System some human intervention is allowed during feature extraction from a latent, e.g. orienting the fingerprint, marking the region of interest, etc. It further outputs a list of candidates that need to be examined by a latent examiner to accept or reject a fingerprint pair as a match.

Although tremendous progress has been made in improving the speed and accuracy of automated fingerprint identification systems (AFIS), most of these systems work well only in scenarios where the matching is performed between rolled or plain fingerprint images. The results of Fingerprint Vendor Technology Evaluation (FpVTE) [18] showed that the most accurate commercial fingerprint matchers achieved an impressive rank-one identification rate of more than $99.4 \%$ on a database of 10,000 plain fingerprint images (see results of Medium Scale Test on page 56 in [18]). On the other hand, the accuracy of latent to rolled print match continues to be quite low. The NIST latent fingerprint testing workshop reported that the rankone accuracy of an automatic latent matcher can be as low as $54 \%$ on a large database of more than 40 million subjects [10]. Currently, NIST is conducting a multi-phase project on Evaluation of Latent Fingerprint Technologies (ELFT) [4]; phase-I results [7] showed that the best identification accuracy obtained is $80 \%$ in identifying 100 latent images among a set of 10,000 rolled prints. This accuracy is still significantly lower than the accuracy of rolled print to rolled print matching on a similar size database.

The difficulty in latent matching is mainly due to three reasons: (i) poor quality of latent prints in terms of the clarity of ridge information, (ii) small area of latent prints as compared to rolled prints and (iii) large non-linear distortion due to pressure variations. Figure 1 shows a latent from the NIST SD27 along with its corresponding rolled print. In Figure 1(c), the ridge information near the center of the image is obscured by the presence of background noise, extraneous markings and other spurious friction ridges surrounding it. Further, while a typical rolled fingerprint has more than 60 minutiae, a typical latent fingerprint may have only 15 usable minutiae [10]. Thus, latent fingerprint identification is a difficult and important problem which needs significant attention.

In this paper we present an algorithm for matching a latent print to a rolled print that achieves accuracy as high as 93.4\% at rank 20 while matching 258 latent images against a background of 2,258 rolled prints. We use 258 latent fingerprint images from the NIST SD27 as query fingerprints. The background database consists of a total of 2,258 rolled print images from SD27 (258 images) and SD4 (2,000 images, one per finger). We use minutiae, orientation field and 
quality map as the features for matching. For latent prints, all the features are currently manually marked whereas for rolled prints features are automatically extracted.

\section{Feature Extraction}

In our system, three types of features are used: minutiae, orientation field and quality map. A minutia consists of five attributes, namely $x, y$, minutiae direction, type and quality, where $x$ and $y$ represent the position of the minutiae. The quality of minutia is defined to have two levels: 0 (unreliable) and 1 (reliable). Orientation field and quality map are obtained by dividing the whole image into blocks of size $16 * 16$ pixels and assigning a single orientation and quality value to each block. We define three quality levels for a block: background (0), reliable (1), unreliable (2). Both reliable and unreliable blocks belong to foreground. A reliable block has good quality with clear ridge structure such that all the minutiae present in that block (if any) can be extracted or marked reliably. An unreliable block is characterized by poor quality where the ridge structure is not clear and hence some minutiae may be missed. However, orientation field can be estimated in an unreliable block.

\subsection{Latent Fingerprint}

Minutiae in latent prints in the SD27 database have been marked by a team of FBI latent examiners. We have manually classified these minutiae as reliable or unreliable. Minutiae near singular points and pairs of minutiae that are spatially close to each other but have nearly opposite directions are categorized as unreliable. Since the chance of an unreliable minutia being missed by an automatic minutiae extraction algorithm is high, they are marked for special treatment in our matching algorithm. In addition to the minutiae provided in the SD27 database, we also mark the orientation field of latents. It is first estimated using a gradient-based algorithm and then modified manually. Due to the poor quality of latents, this process is quite timeconsuming. The quality map of latents is also manually marked. Blocks where ridge structure is clear are marked as reliable blocks whereas blocks where orientation can be reliably estimated but there may be some missing minutiae are marked as unreliable blocks. In addition, blocks around singular points are also classified as unreliable blocks. This is because automatic feature extraction algorithms for rolled prints tend to detect many spurious minutiae in such regions. Figure 2 shows the different types of features marked in a latent image.

\subsection{Rolled Fingerprint}

Rolled fingerprints are automatically processed to obtain minutiae, orientation map and quality map. Our feature extraction algorithm consists of two modules: preprocessing

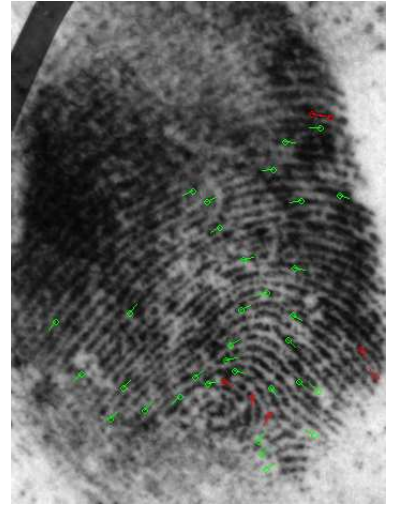

(a)

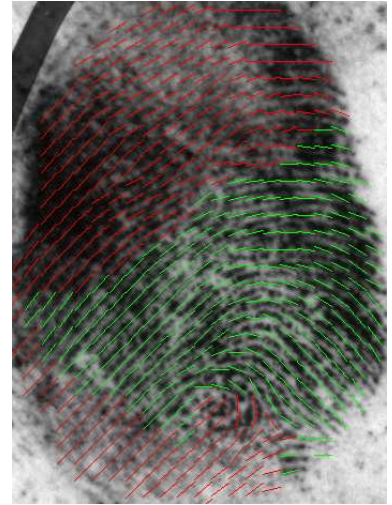

(b)
Figure 2. Features in a latent fingerprint. (a) Minutiae (green: reliable minutiae, red: unreliable minutiae), (b) orientation field and quality map (green: reliable blocks, red: unreliable blocks)

and postprocessing. In this work, Neurotechnology Verifinger 4.2 SDK [2] has been used as a preprocessor. Due to the presence of handwritten characters and strokes on many fingerprints scanned from paper, like the rolled prints in SD4 and SD27, Verifinger produces many false minutiae. Therefore, a postprocessing algorithm was developed to remove these unreliable minutiae and ridges. The quality map is created based on the automatically extracted ridges. We developed a minutiae validation algorithm to classify each minutia as spurious, reliable or unreliable. Ridges are also classified as true ridges or false ridges by a ridge validation algorithm. The results of the various processing steps are shown in Figure 3. In the following paragraphs, we will describe minutia validation, ridge validation, quality map construction and orientation field estimation.

\section{Minutia validation}

A minutia is deemed as spurious if it is close to a background block. A minutia is deemed as unreliable if it forms an opposite pair with other minutia. An opposite pair is a pair of minutiae which are close to each other but have opposite directions. Remaining minutiae are deemed as reliable.

\section{Ridge validation}

It consists of the following two steps. In the first step each ridge is broken into several segments at unreliable ridgels (ridgels are defined as adjoining sequences of six consecutive pixels on a ridge). Reliability of a ridgel is based on neighboring ridge pixels of the two endpoints of the ridgel (see Figure 4). A ridgel is called reliable if: (i) neighboring ridge pixels on both sides are continuous, or (ii) discontinuity in neighboring ridge pixels is caused by a reliable minutia (such as minutia $b$ in Figure 4). In the second step two ridges 


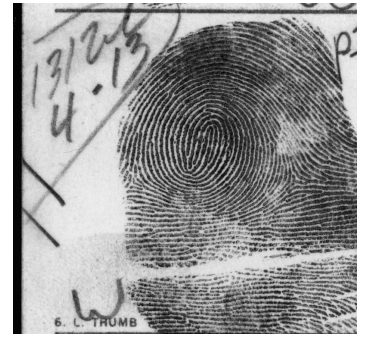

(a)

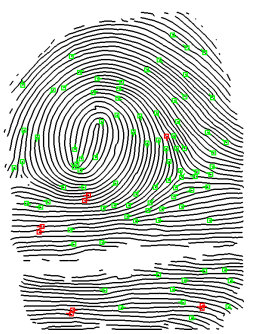

(c)

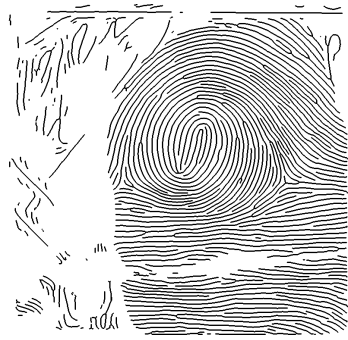

(b)

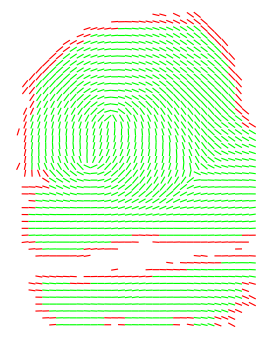

(d)
Figure 3. Images and features of a rolled fingerprint at different stages of feature extraction algorithm. (a) Gray image, (b) Thinning image, (c) Ridges and minutiae (green: reliable minutiae, red: unreliable minutiae), (d) Orientation field and quality map (green: reliable blocks, red: unreliable blocks).

are deemed compatible if there are more than 12 pixels on each ridge that are neighbors of each other. The connected components (ridge groups) are then found using a depth-first search algorithm. A ridge group is deemed reliable, if the number of ridge pixels in this group is greater than a predefined threshold (1,000 in our experiment). All the ridges in a reliable group are deemed as reliable. The unreliable ridges are removed from the image after quality map is created.

\section{Quality map construction}

After ridge extraction, a block containing any ridge pixel is labeled as reliable and other blocks as background blocks. After ridge validation, we set blocks containing unreliable ridges as background. Finally, a reliable block whose 8 neighbors are not all reliable blocks is labeled as an unreliable block.

\section{Orientation field estimation}

For estimating the orientation field, fingerprint image is divided into blocks of size $16 * 16$. For each ridge, its direction is estimated based on ridge points sampled at equal intervals ( 6 pixels). Let $\operatorname{dir}$ be the direction at the $i^{t h}$ point on the ridge in the $(m, n)^{t h}$ block. We accumulate the contributions of horizontal and vertical components of the direction separately in $D X(m, n)$

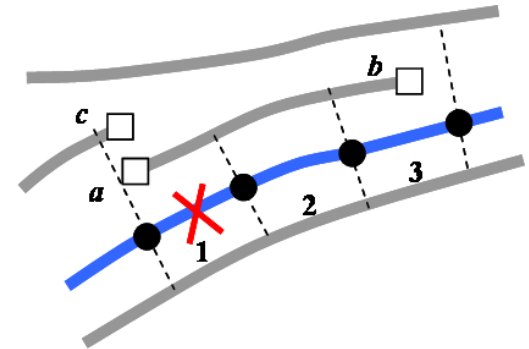

Figure 4. Reliability of ridgel. Ridgel 1 is unreliable due to an unreliable minutia $a$. Both ridgels 2 and 3 are reliable.

and $D Y(m, n)$ as follows:

$$
\begin{aligned}
& D X(m, n)=D X(m, n)+\cos (2 * d i r) \\
& D Y(m, n)=D Y(m, n)+\sin (2 * d i r)
\end{aligned}
$$

After all the ridges have been processed, we compute the orientation field of a block as $\operatorname{atan} 2(D Y, D X) / 2$ $(\operatorname{atan} 2(\mathrm{x}, \mathrm{y})$ returns a value $\theta \in\{-\pi, \pi\}$ such that $\cos (\theta)=x / \sqrt{x^{2}+y^{2}}$ and $\left.\sin (\theta)=y / \sqrt{x^{2}+y^{2}}\right)$.

\section{Matching}

Given the features in a latent fingerprint and a rolled fingerprint, the matching algorithm consists of (i) Local minutiae matching - Similarity between each minutia of latent fingerprint and each minutia of rolled fingerprint is computed. (ii) Global minutiae matching - Using each of the five most similar minutia pairs as an initial minutia pair, a greedy matching algorithm is used to find a set of matching minutia pairs. (iii) Matching score computation - A matching score is computed for each set of matching minutia pairs and the maximum score is used as the matching score between the latent and rolled prints.

\subsection{Local Minutiae Matching}

In this step, the similarity between each minutia of latent fingerprint and each minutia of rolled fingerprint is computed. Since the basic properties of a minutia, like location, angle and type, are not very distinctive features, additional information, which is referred to as descriptor [11], is attached to a minutia to make it distinctive. While the matching algorithm in [11] which utilizes minutiae descriptors obtained good results on FVC2002 databases, it has to be adapted to handle latent fingerprints. In this paper, we use two types of descriptors: orientation-based and neighboring minutiae-based (See Figure 5). The similarity between two minutiae is defined as the mean value of the similarity of two types of descriptors.

\section{Orientation-based descriptor}




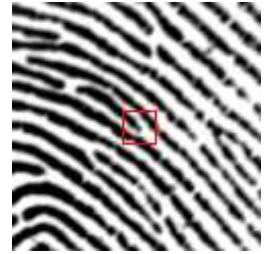

(a)

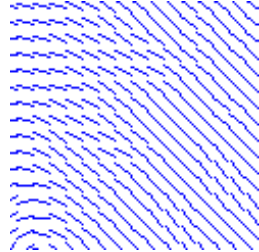

(b)

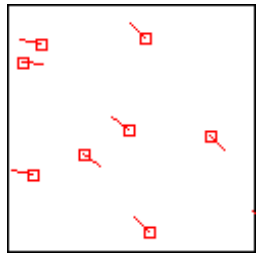

(c)
Figure 5. Minutia descriptor. (a) Local image, (b) orientation field, (c) neighboring minutiae.

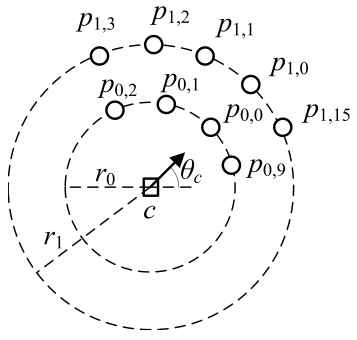

Figure 6. The configuration of orientation descriptor.

For every minutia, a local coordinate system is defined with the minutia as the origin and its direction as the positive $x$ axis. A set of fixed sampling points is defined (See Figure 6) and the local ridge orientation at these sampling points form the orientation descriptor. These sampling points are located on 4 circles centered at the minutia, and distributed equally on each circle. The radii of circles are 27, 45, 63 and 81 and the numbers of sampling points on these circles are $10,16,22$, and 28 , respectively. These parameters have been determined empirically in [17]. The similarity of two orientation descriptors is computed as the mean value of the similarity of all valid sampling points (a sampling point falling in the background region is deemed as invalid). The similarity between the orientations of two sampling points is computed as $s_{p}=\exp (-\operatorname{angle} /(\pi / 16))$, where angle denotes the angle between two orientations. If the number of common valid sampling points is less than $25 \%$ of the total number of sampling points, the similarity of two orientation descriptors is set to 0 .

\section{Neighboring minutiae-based descriptor}

The neighborhood of a minutia is defined to be a circular region of radius 80 pixels. All minutiae lying in this neighborhood are called the neighboring minutiae. Let $p$ and $q$ be the two minutiae whose similarity is to be computed. For each neighboring minutia $p_{i}$ of $p$, we examine if there is a neighboring minutia of $q$ whose properties (See Figure 7) are similar to those of

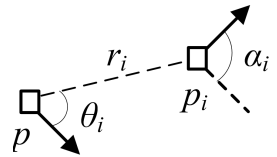

Figure 7. Properties of neighboring minutia $p_{i}$ in the local polar coordinate system defined by minutia $p$.

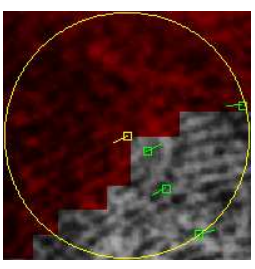

(a)

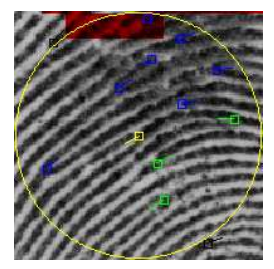

(b)
Figure 8. Comparison of neighboring minutiae based descriptors for two minutiae (shown in yellow) in (a) and (b). (a) All 4 neighboring minutiae (inside the yellow circle) are matched (shown in green), (b) only 3 neighboring minutiae are matched but the others (shown in blue) are not penalized because they correspond to unreliable blocks in (a). Unreliable region is shown in red.

$p_{i}$. If such a minutia exists, $p_{i}$ is deemed as a matching minutia; otherwise $p_{i}$ is checked against the following three criteria: (i) the minutia is unreliable, (ii) it falls in a background region when mapped to the other fingerprint based on the alignment parameters between $p$ and $q$, (iii) it falls in an unreliable block and its direction after being mapped is consistent with the local orientation in the other fingerprint. If $p_{i}$ belongs to any one of the three cases, it will not be penalized; otherwise, it will be penalized. The above process is also applied to the neighboring minutiae of $q$. Then the similarity of neighboring minutiae-based descriptors is computed as

$$
s_{m}=\frac{m_{p}+1}{m_{p}+n_{p}+3} \times \frac{m_{q}+1}{m_{q}+n_{q}+3},
$$

where $m_{p}$ and $m_{q}$ denote the number of matching neighboring minutiae of $p$ and $q$, and $n_{p}$ and $n_{q}$ denote the number of penalized unmatched neighboring minutiae of $p$ and $q$. The example in Figure 8 shows that the similarity between descriptors of two matching minutiae can be improved by not penalizing the unmatched minutiae belonging to either of the above three cases. It should be noted that $m_{p}$ may be different from $m_{q}$ since we do not establish a one-to-one correspondence between minutiae.

\subsection{Global Minutiae Matching}

Given similarity of all minutia pairs, the one-to-one correspondence between minutiae is established in the global 
minutiae matching stage. Greedy strategy is used to find matching minutia pairs in decreasing order of similarity. In order to give priority to those minutia pairs that are not only similar to each other but also dissimilar with other minutiae, a normalized similarity measure $s_{n}$ is defined based on similarity $s$ as:

$$
s_{n}(i, j)=\frac{\left(M_{L}+M_{R}-1\right) * s(i, j)}{\sum_{k=1}^{M_{R}} s(i, k)+\sum_{k=1}^{M_{L}} s(k, j)-s(i, j)},
$$

where $s(i, j)$ denotes the similarity between minutia $i$ and minutia $j$, and $M_{L}$ and $M_{R}$ denote the number of minutiae in the latent and rolled, respectively.

All minutia pairs are sorted in decreasing order of normalized similarity and each of the top 5 minutia pairs is used to align the two sets of minutiae. Minutiae are examined according to decreasing order of their similarity and minutiae that are close in both location and direction, and have not been matched to other minutiae are deemed matching minutiae. After all the minutia pairs have been examined, a set of matching minutiae is returned.

\subsection{Matching Score Computation}

The matching score $S$ between latent and rolled fingerprints is computed as

$$
S=W_{m} * S_{m}+\left(1-W_{m}\right) * S_{o},
$$

where $S_{m}$ and $S_{o}$ denote the matching score based on minutiae and that based on orientation fields, and the weight $W_{m}$ is set empirically as 0.8 .

We align the two fingerprints in order to obtain the orientation field matching score in the following manner. Let $(\Delta x, \Delta y, \Delta \theta)$ be the parameters of the geometric transformation between the latent and the rolled print. The rotation angle $\Delta \theta_{i}$ is computed between the $i$ th pair of matching minutiae, $i=1, \cdots, M$, where $M$ denotes the number of matching minutiae. Then the average rotation angle $\Delta \theta$ is computed as

$$
\Delta \theta=\operatorname{atan} 2\left(\sum_{i=1}^{M}\left(\sin \left(\Delta \theta_{i}\right)\right), \sum_{i=1}^{M}\left(\cos \left(\Delta \theta_{i}\right)\right)\right)
$$

Then $\Delta x$ and $\Delta y$ are computed as

$$
\begin{aligned}
& \Delta x=\frac{1}{M} \sum_{i=1}^{M}\left(x_{R, i}-x_{L, i} \cos (\Delta \theta)-y_{L, i} \sin (\Delta \theta)\right) \\
& \Delta y=\frac{1}{M} \sum_{i=1}^{M}\left(y_{R, i}+x_{L, i} \sin (\Delta \theta)-y_{L, i} \cos (\Delta \theta)\right),
\end{aligned}
$$

where $x_{L, i}, y_{L, i}, x_{R, i}$ and $y_{R, i}$ denote the coordinates of the $i^{\text {th }}$ pair of matching minutiae and the first subscript denotes latent or rolled.
The matching score $S_{o}$ based on orientation field is the product of a quantitive score $S_{o n}$ and a qualitative score $S_{o q}$. The quantitive score $S_{o n}$ is computed as $N_{b} /\left(N_{b}+\right.$ $100)$, where $N_{b}$ is the number of blocks where the difference of orientation in latent and rolled print is less than $\pi / 8$. The qualitative score $S_{o q}$ is computed as $\left(1-2 * D_{o} / \pi\right)$, where $D_{o}$ is the mean of the difference of orientation values of all the blocks.

The matching score $S_{m}$ based on minutiae is also the product of a quantitive score $S_{m n}$ and a qualitative score $S_{m q}$. The quantitive score $S_{m n}$ is computed as $M /(M+8)$. The qualitative score is computed as

$$
S_{m q}=S_{d} \times \frac{M}{M+N_{L}} \times \frac{M}{M+N_{R}},
$$

where $S_{d}$ is the average similarity of descriptors for all the matching minutiae, and $N_{L}$ and $N_{R}$ denote the number of penalized unmatched minutiae (defined in Section 3.1) in latent and rolled prints, respectively.

\section{Experimental Results}

The experiments were conducted on NIST SD27 database [10] which contains 258 latent fingerprints and their corresponding rolled prints at 500 ppi. This is the only public domain database available containing mated latent and rolled prints. These 258 latent prints were classified by latent examiners into three classes, namely: Good, Bad and Ugly based on the quality. There are 88 "Good", 85 "Bad" and 85 "Ugly" latent images in the database. Since there are only 258 rolled fingerprints in SD27, we expand the background database by adding fingerprints from NIST SD4. There are 2,000 different fingers and 2 rolled impressions per finger in SD4. These fingerprints were also scanned from paper and have similar characteristics to the rolled prints in SD27. One rolled fingerprint for each finger in SD4 is taken in addition to the rolled images in SD27 to form a background database containing 2,258 rolled prints.

Since marking additional features like orientation field and quality map in latents will increase the workload of latent examiner, it is desirable to know how much performance gain can be obtained by using these additional features. Therefore, we also have a simplified version of the proposed matching algorithm that uses only minutiae. The original matching algorithm and the simplified version are referred to as $\mathrm{M}$ algorithm and MS algorithm, respectively. Figure 9 shows the Cumulative Match Characteristic (CMC) curves for both the algorithms tested on small sized background database (258 rolled prints) and medium sized background database (2,258 rolled prints). It can be observed that using additional features improves the accuracy significantly, especially on the medium sized database. M algorithm reaches a rank-1 identification rate of $79.5 \%$ and a rank-20 identification rate of $93.4 \%$ on the medium sized 
database. It should be noted that, in practice, latent examiners will check each of the candidates returned by an AFIS to find the correct match. The number of candidates is generally 20 or 30 for medium sized databases and 100 for large sized databases like IAFIS. Therefore the rank- 20 rate is a more useful measure than the rank-1 rate on the medium sized database.

Singular points have been used to speedup fingerprint matching algorithms and filter the background database. To examine the effect of singular points, we used the northmost core points in SD27, which are marked by experts, to coarsely align fingerprints before using $\mathrm{M}$ algorithm. It was observed that use of core points improved the rank-1 rate from $86.4 \%$ to $91.1 \%$ on the small sized background database.

Figure 10 shows the CMC curves of the $\mathrm{M}$ algorithm separately for good, bad and ugly quality latent prints. The medium sized background database is used. As expected, the matching performance for good quality latents is significantly better than that for the latents belonging to the other two quality groups. Three examples of successful identification (one from each quality group) are shown in Figure 11. The mated rolled print was found at rank 1 by algorithm M. It should be noted that although there are only 4 matching minutiae in the ugly latent (Figure 11(c)), our algorithm still identified it correctly. However, if a large number of spurious minutiae are detected in the common (overlapping) region of latent and rolled prints, the matching algorithm will fail as shown in Figure 12.

To our knowledge, only ELFT07 [4] has reported matching performance on SD27. ELFT07 tested fully automated latent search technology by searching 100 latents against a background database of 10,000 rolled prints. But, only average performance over all ten participating vendors is reported in [4]. The average rank-1 rate is about $60 \%$ on background of 10,000 rolled prints and $67 \%$ on background of 1,000 rolled prints. The results of ELFT07 and our results can not be compared directly. In ELFT07, out of 100 latents only 50 are from SD27, and the quality of these selected latents is unknown. As shown in Figure 10, the accuracies for different quality latents are significantly different.

The experiments were conducted on a PC with Intel Core 2 Duo CPU and Windows XP operating system. The automatic feature extraction takes $572 \mathrm{~ms}$ for a rolled print in NIST SD4 and $723 \mathrm{~ms}$ for a print in NIST SD27. It takes a total of 30 seconds to match a latent against all the 2,258 rolled prints.

\section{Summary and Discussion}

We have proposed a system for matching latent fingerprints with rolled fingerprints. In addition to minutiae, orientation field and quality map are also used as features. A minutiae-based fingerprint matching algorithm is devel-

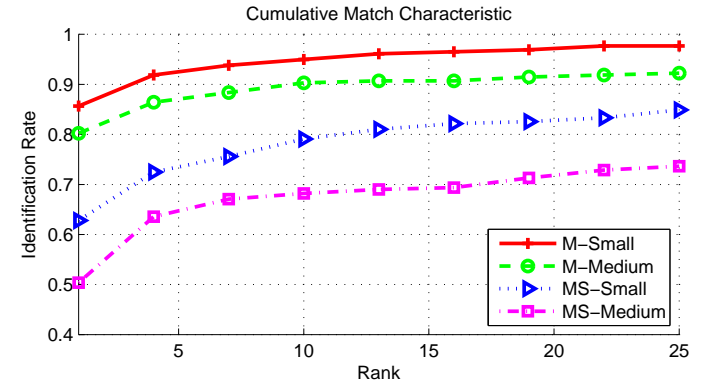

Figure 9. CMC curves of two algorithms (M and MS) on small sized and medium sized background databases (258 and 2,258 rolled prints).

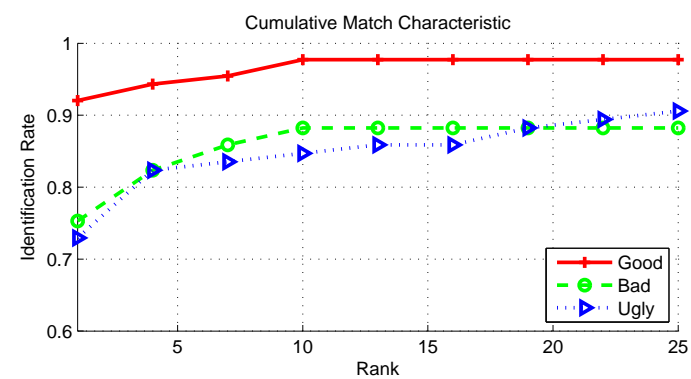

Figure 10. CMC curves corresponding to Good, Bad and Ugly quality latent images in the NIST SD27 database. These three categories contain 88,85 and 85 latent images, respectively. The medium sized background database (2,258 rolled prints) and $\mathrm{M}$ algorithm are used.

oped. Both level-1 and level-2 features are considered in computing matching scores, and at each feature level both quantitive scores and qualitative scores are computed. To test the proposed system, 258 latent fingerprints in NIST SD27 are matched against a background database that consists of 258 rolled fingerprints in SD27 and 2,000 rolled fingerprints in SD4. Our system reaches a rank-1 identification rate of $79.5 \%$ and a rank-20 identification rate of $93.4 \%$. We have also shown empirically that use of additional information, like orientation field and quality map, improves the matching accuracy significantly.

Considering the importance of singular points, we plan to develop a robust singular point detection algorithm and use singular points to improve the speed and accuracy of the current system. To make the latent fingerprint matching system operate in a lights-out mode, automatic feature extraction from latent fingerprints is necessary. As a future work, automatic extraction of level-1 and level-2 features from latent fingerprints will be explored. We will also investigate how much performance gain can be obtained by using extended features [3], e.g. incipient ridges, dots and pores, in latent fingerprint matching. When the background database contains millions of fingerprints, some speedup 

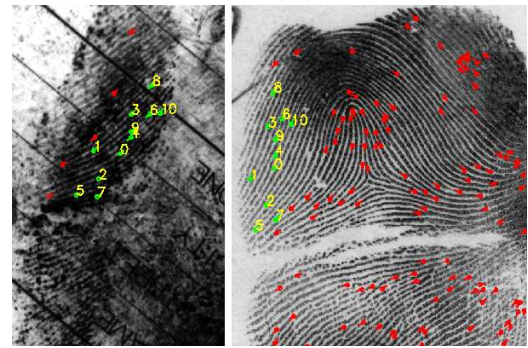

(a)
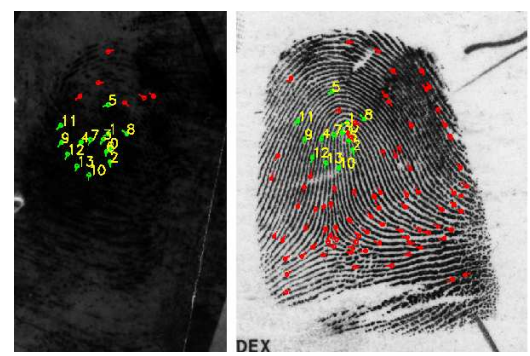

(b)
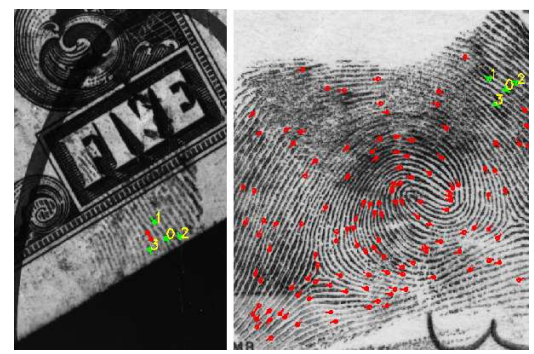

(c)

Figure 11. Examples of successful matching. Three latents (classified as good, bad and ugly by latent examiner) and the mated rolled prints. In all these three cases, our algorithm found the true mate at rank one
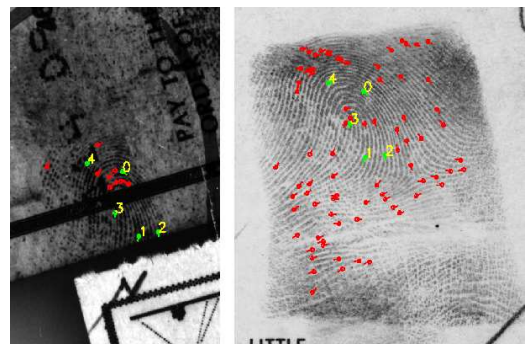

Figure 12. Example of an incorrect match. The mated rolled print (right) of the latent (left) was ranked 245 by our algorithm. Many spurious minutiae are detected in the rolled print.

technique will be necessary. We plan to design an indexing system which will filter out a large portion of the background database quickly and reliably.

\section{References}

[1] Case Profile, Innocence Project. http://www . innocenceproject.org/Content/73.php.

[2] Neurotechnology Inc., Verifinger. http://www. neurotechnology.com.

[3] CDEFFS: the ANIS/NIST Committee to Define an Extended Fingerprint Feature Set. http: / / ingerprint.nist. gov/standard/cdeffs/index.html.

[4] Evaluation of latent fingerprint technologies 2007. http: //fingerprint.nist.gov/latent/elft07/.

[5] A review of the FBI's Handling of the Brandon Mayfield Case. Office of the Inspector General, Special Report, March 2006. http://www.usdoj.gov/oig/ special/s0601/PDF_list.htm.

[6] Conclusion of circuit court judge Susan Souder - grants motion to exclude testimony of forensic fingerprint examiner capital murder case: State of Maryland v. Bryan Rose, October 2007. http: / / www.clpex.com/Information/ STATEOFMARYLAND-v-BryanRose.doc.

[7] Summary of results from ELFT07 phase I testing, September 2007. http://fingerprint.nist.gov/latent/ elft07/phase1_aggregate.pdf.

[8] D. Ashbaugh. Quantitative-Qualitative Friction Ridge Analysis: Introduction to Basic Ridgeology. CRC Press, 1999.

[9] P. M. Christophe Champod, Chris Lennard and M. Stoilovic, editors. Fingerprints and Other ridge Skin Impressions. CRC Press, 2004.

[10] V. N. Dvornychenko and M. D. Garris. Summary of NIST latent fingerprint testing workshop. NISTIR 7377, November 2006. http://fingerprint.nist.gov/latent/ ir_7377.pdf.

[11] J. Feng. Combining minutiae descriptors for fingerprint matching. Pattern Recognition, 41(1):342-352, 2008.

[12] P. Komarinski, editor. Automated Fingerprint Identification Systems (AFIS). Elsevier Academic Press, 2001.

[13] H. C. Lee and R. E. Gaensslen, editors. Advances in Fingerprint Technology. CRC Press, New York, 2001.

[14] D. Maltoni, D. Maio, A. K. Jain, and S. Prabhakar. Handbook of Fingerprint Recognition. Springer-Verlag, 2003.

[15] A. A. Moenssens. Is fingerprint identification a science? Forensic-Evidence.com, 1999. http: //forensic-evidence.com/site/ID/ID00004_ 2. html.

[16] M. J. Saks and J. J. Koehler. The coming paradigm shift in forensic identification science. Science, 309, August 2005.

[17] M. Tico and P. Kuosmanen. Fingerprint matching using an orientation-based minutia descriptor. IEEE Transactions on Pattern Analysis and Machine Intelligence, 25(8):10091014, 2003.

[18] C. Wilson. Fingerprint vendor technology evaluation 2003: Summary of results and analysis report. NISTIR 7123, June 2004. http: / / fpvte.nist.gov/report/ir_ 7123_analysis.pdf. 